\title{
Diagramas de Voronoi para a definição de áreas de abrangência de hospitais públicos no Município do Rio de Janeiro
}

\author{
Defining catchment areas for public hospitals \\ in the Municipality of Rio de Janeiro through \\ Weighted Voronoi Diagrams
}

Flavio Astolpho Vieira Souto Rezende 1

Renan M.Varnier Almeida 2

Flavio F. Nobre 2

\footnotetext{
1 Centro Municipal de Saúde Manoel José Ferreira, Secretaria Municipal de Saúde do Rio de Janeiro. Rua Silveira Martins 161, Rio de Janeiro, $R J$ 20000-000, Brasil. 2 Programa de Engenharia Biomédica, Coordenação dos Programas de Pós-graduação e Pesquisa em Engenharia, Universidade Federal do Rio de Janeiro. Cidade Universitária, C. P. 68510 , Rio de Janeiro, $R J$ 21945-970, Brasil.
}

\begin{abstract}
One of the most important pieces of information for health resources planning is the definition of catchment areas for health units. Voronoi Diagrams are a potential technique for this purpose. They are polygons with the property whereby adjacent polygons have their borders located within the same distance of the respective generator points. One possible adjustment to the catchment areas thus defined is the use of weighted Voronoi Diagrams, which result in an improved representation of a health unit's actual capacity. In this study, the 21 public general hospitals in the city of Rio de Janeiro, Brazil, were used as generator points for Voronoi Diagrams. Non-weighted Voronoi Diagrams were initially implemented and then used as the basis for obtaining weighted Voronoi Diagrams, using as weights the annual admission rates estimated for each unit. In the classic Voronoi Diagram case, some catchment areas had similar sizes, although their respective health units had different characteristics. In the weighted case the areas were modified in a way that appeared closer to the actual functioning of the units. The method appeared simple to implement, used easy-to-access data, and did not rely on geopolitical considerations such as existing administrative areas. It thus provided a more realistic picture of a unit's capacity to support basic health programs.
\end{abstract}

Key words Accessibility; Voronoi Diagrams; Health Planning

Resumo No planejamento de recursos em saúde é importante o conhecimento da área de abrangência de uma unidade. Os Diagramas de Voronoi constituem uma técnica para tal; são polígonos construídos de tal forma que as bordas de polígonos adjacentes encontram-se eqüidistantes de seus respectivos pontos geradores. Uma modificação nas áreas de abrangência assim definidas é sua ponderação (Diagramas de Voronoi ponderados), representando a capacidade da unidade de forma mais real. No presente trabalho foram utilizados, como pontos geradores, 21 hospitais gerais públicos no Rio de Janeiro, RJ. Inicialmente foram criados os Diagramas de Voronoi sem ponderação, e, a partir destes, os diagramas ponderados, empregando-se como variável de ponderação as estimativas de internação anual para cada unidade. Na divisão clássica, áreas de abrangência similares foram atribuídas a hospitais com características diferenciadas, problema esse contornado no método ponderado. O método é de simples implementação e visualização, utiliza dados de fácil acesso e independe de parâmetros arbitrários ou geopolíticos. Portanto, esses diagramas podem fornecer, a gerentes de saúde, uma visão mais realista para o planejamento da demanda de suas unidades.

Palavras-chave Acessibilidade; Diagramas de Voronoi; Planejamento em Saúde 


\section{Introdução}

A acessibilidade a uma unidade de saúde deve ser consideração importante para órgãos gerenciadores, em virtude de este constituir um aspecto fundamental para garantir a uma população, pelo menos, o atendimento primário à saúde. Para que isto ocorra, é essencial que exista igualdade na distribuição dos recursos da saúde, bem como que essa seja a primeira preocupação dos setores governamentais (Zaidi, 1994). Ao mesmo tempo, a pressão de recursos limitados faz com que muitos estudos estejam atualmente voltados a facilitar o acesso às unidades de saúde e, em conseqüência, a reduzir custos e otimizar o uso do equipamento de saúde pela população (Salmela, 1993).

No Brasil, com a promulgação da Lei 8.080 em 19 de setembro de 1990, foi criado o Sistema Único de Saúde (SUS), o qual passa a atender toda a população, independente de seu vínculo previdenciário, utilizando, para tal, os serviços de saúde do sistema público - serviços estatais e serviços privados contratados pela Previdência Social: lucrativos ou filantrópicos (Giovanella \& Fleury, 1996). Apesar dessas modificações, o acesso às unidades de saúde continua precário em muitas cidades do Brasil, em especial, devido a problemas até agora não resolvidos de financiamento do SUS, o que gera diminuição de sua oferta de serviços.

Além do aspecto de localização geográfica, a questão do acesso a unidades tem sido analisada também em termos dos fatores que limitam o deslocamento de um paciente a determinada unidade (Unglert et al., 1987; Unglert, 1990; Love \& Lindquist, 1995). A dificuldade no acesso a uma unidade de saúde pode apresentar vários motivos, como, por exemplo, obstáculos geográficos - morros, estradas, rios -; obstáculos sociais, caso de unidades localizadas em comunidades fechadas - quartéis, presídios -; e de atendimento, como o que ocorreu por bom tempo, no Brasil, à época do surgimento da assistência previdenciária (Oliveira \& Teixeira, 1986). A definição da área de abrangência de uma unidade, e, por conseguinte, de sua população adscrita, é fundamental para tratamento mais amplo deste problema, na tentativa de planejar os recursos a serem alocados em cada região. Na maioria das vezes, não é possível estabelecer claramente essa área, podendo ser utilizada a população contida em um distrito de saúde ou sanitário, ou, como no caso do município do Rio de Janeiro, em Áreas de Planejamento (AP) usadas pela Secretaria Municipal de Saúde do Rio de Janeiro (SMS-RJ).
Existem várias metodologias que servem de auxílio na definição dessas áreas de abrangência, algumas que exigem recursos matemáticos e estatísticos complexos, dificultando sua utilização por gerências na saúde. Com o desenvolvimento de programas de computação que permitem análises estatísticas mais aprofundadas, tornou-se possível realizar também estudos de distribuição, localização e regionalização das unidades de saúde (Love \& Lindquist, 1995; Bullen et al., 1996; Kohli et al., 1997). O impulso para que a análise geográfica computadorizada se tornasse campo multidisciplinar ocorreu durante os anos 70, mas ainda com raízes profundas na matemática cartográfica. Este impulso foi ampliado pelas facilidades implantadas para o uso de computadores e com a produção de Sistemas de Informações Geográficas (SIG), que contam com interfaces "amigáveis", as quais necessitam de menor conhecimento de programação por parte do operador e permitem a análise e representação de dados geograficamente referenciados. A partir daí, houve igualmente estímulo ao desenvolvimento de novas tecnologias, empregadas nas diferentes fases do planejamento dos sistemas de informação (Nijkamp \& Scholten, 1993; Clarke et al., 1996).

O presente trabalho irá ater-se a sugerir às gerências de saúde, em todos os níveis, um método para visualizar a divisão de um município, o Diagrama de Voronoi Generalizado. Serão utilizados, como centróides desses diagramas, hospitais gerais públicos do Município do Rio de Janeiro, RJ. Após a obtenção desses polígonos - e estando disponíveis os setores censitários localizados em cada polígono - torna-se possível a obtenção da população adscrita a cada unidade de saúde indicada como centróide. Os fundamentos do método e os resultados de sua aplicação são discutidos a seguir.

\section{Diagrama de Voronoi}

O Diagrama de Voronoi - igualmente chamado Polígono de Thiessen ou Tesselação de Delaunay - é empregado nas áreas de arqueologia, biologia, cartografia, mineralogia, meteorologia, fisiologia, estatística e, mais recentemente, em planejamento urbano e regional (Boots et al., 1995). A história do uso do Diagrama de Voronoi inicia-se com Descartes, em dois de seus trabalhos, "Le Monde de Mr. Descartes" e "Le Traité de La Lumiére”, publicados em 1644, e no terceiro capítulo do livro Principia Philosophiae. Esses diagramas, usados para demonstrar a disposição da matéria no sistema solar, são semelhantes aos criados posteriormente por Voronoi. 
Os primeiros trabalhos que efetivamente apresentaram polígonos na forma do diagrama são os de Dirichlet, realizado em 1850, e o de Voronoi, em 1908, os quais, ao estudarem formas quadráticas, consideraram uma forma especial do Diagrama de Voronoi. A diferença na abordagem desses trabalhos é que Dirichlet estudava o diagrama em duas e três dimensões, enquanto Voronoi o estudava em $n$ dimensões (Boots et al., 1995). No entanto, somente em 1911 foram iniciados estudos, por um pesquisador da área de meteorologia de nome Thiessen, que usavam os Diagramas para a representação espacial do relacionamento entre áreas geográficas. Em seu trabalho, Thiessen empregou o Diagrama de Voronoi - denominando-o Polígono de Thiessen - para estimar com mais acurácia as médias regionais de precipitação de chuva (Rhynsburger, 1973; Brassel $\&$ Reif, 1979). A partir desse momento, sua aplicação passou a ser mais intensa tanto na área de meteorologia como em outras, por exemplo, cartografia e planejamento urbano (Sibson, 1980; Martin \& Willams, 1992). Este incremento ocorreu também em decorrência das facilidades de processamento introduzidas pela informática e pela construção de algoritmos, como o de Green \& Sibson (1977) e o de Brassel \& Reif (1979), que possibilitaram a criação de polígonos de forma mais rápida e em $n$ dimensões. Após isso, outros algoritmos surgiram, como os apresentados por Bowyer (1981), Watson (1981), Macedonio \& Pareschi (1991) e Tsai (1993).

$\mathrm{Na}$ área de planejamento em saúde há insuficientes aplicações do método. Um dos poucos exemplos é o estudo de Zwarestein et al. (1991), na África do Sul, que analisou o efeito, no acesso aos hospitais, da disponibilização de leitos hospitalares para todos, indiferente da cor, negro ou branco. Para calcular tal efeito, foi preciso definir áreas de abrangência para cada hospital estudado. Neste ponto da pesquisa tornou-se necessário o uso de Diagramas de Voronoi, através do uso de um SIG, o ArcInfo, que permite o cálculo do diagrama. Na pesquisa pôde-se observar que, nas áreas rurais, a abertura dos leitos não alterou o acesso aos hospitais de modo significativo em decorrência dos poucos leitos oriundos de hospitais para brancos. Já na zona urbana foi detectado pequeno efeito, mas ainda sem resolver a disparidade de leitos por habitantes, o que evidenciava maior necessidade de leitos.

A construção do Diagrama de Voronoi ocorre primeiramente ao tomar-se em conta um conjunto de pontos não colineares, $\mathrm{P}=\left\{\mathrm{p}_{1}\right.$, $\left.\mathrm{p}_{2}, \ldots, \mathrm{p}_{\mathrm{n}}\right\}, \mathrm{n}>2$, no plano Euclidiano, denominados “pontos geradores". Entre esses pontos, são traçadas retas eqüidistantes entre si, que irão formar as bordas dos polígonos. Cada polígono, fechado e adjacente a outro, contendo apenas um dos pontos geradores, é denominado Diagrama de Voronoi, conforme ilustrado na Figura 1 (Rhynsburger, 1973; Boots et al., 1995).

No Diagrama de Voronoi Clássico, os pontos geradores possuem o mesmo peso e sua construção depende apenas da distância entre eles. No atual trabalho optou-se por utilizar uma generalização do método, designada como Diagrama de Voronoi Ponderado. Nessa forma, os pontos geradores podem assumir pesos diferenciados, o que irá transformar as bordas dos polígonos, de retas, em arcos de circunferências ou partes de arcos. Assim, obtêm-se polígonos não só proporcionais a suas relações geográficas, mas também ao poder de atração de cada um deles.

No presente trabalho utilizou-se uma fórmula apresentada por Boots (1980) para calcular o Diagrama de Voronoi Ponderado.

Após o traçado de todas as circunferências referentes a um ponto gerador e seus vizinhos, faz-se necessário estudar sua "dominância” e "contradominância". Para isso definiu-se como dominância, no traçado dos Diagramas, a área resultante da superposição dos polígonos produzidos por dois hospitais adjacentes - que é atribuída como adscrita àquele hospital estudado - e, como contradominância, o complemento desta área, considerada adscrita ao primeiro hospital, ou seja:

Figura 1

Diagrama de Voronoi

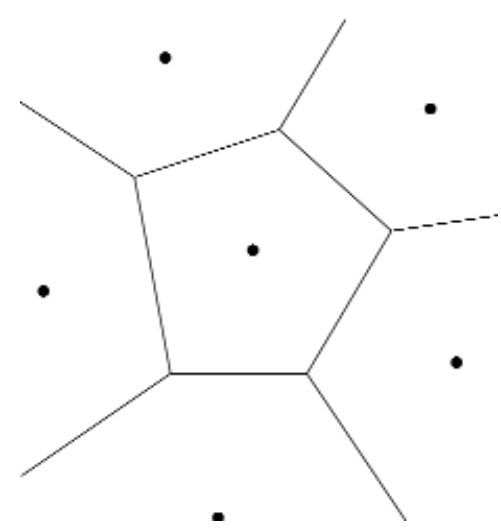

Cad. Saúde Pública, Rio de Janeiro, 16(2):467-475, abr-jun, 2000 
$\operatorname{Dom}_{\mathrm{A} \rightarrow \mathrm{B}}=$ Área da circunferência que possui A como ponto gerador - Área da circunferência que possui $\mathrm{B}$ como ponto gerador.

Onde,

$\mathrm{A}=$ ponto estudado.

$\mathrm{B}=$ ponto vizinho e comparado a A.

Propostas de algoritmos para Diagramas de Voronoi ponderados podem igualmente ser encontradas em Boots (1980), Boots et al. (1995), Lee \& Drysdale (1981) e Aurenhammer \& Edelsbrunner (1984).

\section{Materiais e métodos}

\section{Definição do Diagrama de Voronoi}

Os pontos geradores utilizados no trabalho foram os hospitais gerais públicos do Município do Rio de Janeiro, RJ, assim definidos pela Fundação Instituto Brasileiro de Geografia e Estatística (IBGE). O IBGE define como hospital aquele estabelecimento que tem por finalidade básica prestar assistência médica em regime de internação por período mínimo de 24 horas, possuindo leitos e instalações apropriadas, além de assistência permanente de, pelo menos, um médico. A definição de unidade geral é a de "estabelecimento capacitado a prestar assistência de saúde, com ou sem internação elou que tenha condições de atendimento nos quatros serviços básicos, definidos como: clínica médica, clínica cirúrgica, clínica gineco-obstétrica e clínica pediátrica". Em relação à definição de unidade pública este é o "estabelecimento onde não existe restrição de atendimento à população" (IBGE, 1994). "População adscrita" de uma unidade pode ser definida como a população situada dentro de seu raio de ação. Empregando-se essas definições e tomando-se em conta que as unidades deveriam possuir pelo menos dois dos serviços básicos citados na definição de unidade geral, no tocante a leitos de internação, foram determinadas 21 unidades, localizadas no município do Rio de Janeiro (Tabela 1). No caso do Hospital Universitário Clementino Fraga, foi considerado como sua parte integrante o Instituto de Puericultura e Pediatria Martagão Gesteira, localizado em prédio anexo.

\section{Construção dos Diagramas de Voronoi}

Uma vez definida geograficamente a localização dos 21 pontos geradores, procedeu-se à construção dos polígonos. Opções para sua construção podem ser encontradas em vários “pacotes”, como, por exemplo, o SIG SpanMap.
Como o objetivo da pesquisa incluía também a construção de polígonos ponderados, fez-se necessário o uso de outra variável para que cada ponto (hospital) recebesse peso diferenciado de acordo com características relevantes, as quais supõe-se que podem alterar seu "poder de atração". Muitas variáveis podem ser empregadas para isso, como, por exemplo, número de profissionais de saúde lotados na unidade, número de leitos disponíveis na unidade, capacidade instalada ou sua oferta anual de internações. Optou-se por usar, como variável ponderada, a oferta anual de internações de cada um dos hospitais selecionados.

Tal oferta de internações foi avaliada com base no anexo à Portaria do Ministério da Previdência e Assistência Social (MPAS), de número 3046/82 (MS, 1982), que tem como título " $\mathrm{Pa}$ râmetros para planejamento assistencial a serem utilizados no INAMPS" e que é, além disso, muito usada no planejamento em Saúde, apesar de ser portaria relativamente antiga (1982). Por intermédio desta portaria, admitindo-se que todas unidades obtiveram taxa de ocupação igual a $80 \%$ e tempos médios de permanência iguais a 6,5 dias (Clínica Médica), 5,8 dias (Clínica Cirúrgica), 3,7 dias (Clínica Obstétrica) e 36,5 dias (Clínica Psiquiátrica), o número de internações oferecido por clínica pode ser calculado através dos seguintes valores: Clínica Médica: 45 internações/leitos/ano; Clínica Cirúrgica: 50 internações/leitos/ano; Clínica Obstétrica: 80 internações/leitos/ano; Clínica Psiquiátrica: 8 internações/leitos/ano.

Para efeitos de cálculo, a Clínica Pediátrica foi incluída na Clínica Médica; a Clínica Ginecológica, na Clínica Cirúrgica; e os leitos de Tisiologia e Fora de Probabilidade Terapêutica foram incluídos na Clínica Psiquiátrica. Obteve-se o número de leitos no cadastro do Sistema de Informação Hospitalar, SIH/SUS, de janeiro de 1997, que se encontra disponível em CDROM, pela Internet e pelo BBS do Ministério da Saúde (MS-BBS) (MS, 1997).

A Tabela 2 mostra o número total de internações, relativo a cada unidade de saúde selecionada, possíveis de serem oferecidas, em um ano, por hospital. A partir desses dados, o percentual de cada unidade foi calculado sobre o total de internações oferecidas no município, recebendo este valor a denominação de "peso" (W), ou seja:

$\mathrm{W}=$ peso $=\frac{\text { número de internações s estimadas para a unidade }}{\text { total de intenções s estimadas para o município }} * 100$

Foram então calculadas as coordenadas e raios dos novos Diagramas de Voronoi, tomando-se em conta as vizinhanças determinadas 
Hospitais utilizados como pontos geradores dos Diagramas do Voronoi, seus códigos e bairros de localização, Município do Rio de Janeiro.

\begin{tabular}{cll}
\hline Código & \multicolumn{1}{c}{ Unidade } & Bairro em que está localizado \\
\hline 1 & Hospital Estadual Pedro II & Santa Cruz \\
2 & Hospital Estadual Rocha Faria & Campo Grande \\
3 & Hospital Estadual Albert Schweitzer & Bangu \\
4 & Hospital Raphael de Paula Souza & Jacarepaguá \\
5 & Hospital Estadual Carlos Chagas & Marechal Hermes \\
6 & Hospital Municipal Lourenço Jorge & Barra da Tijuca \\
7 & Hospital Geral de Jacarepaguá & Jacarepaguá \\
8 & Hospital Geral da Piedade & Piedade \\
9 & Hospital Estadual Getúlio Vargas & Penha \\
10 & Hospital Municipal Salgado Filho & Méier \\
11 & Hospital Geral do Andaraí & Andaraí \\
12 & Hospital Geral de Bonsucesso & Bonsucesso \\
13 & Hospital Universitário Pedro Ernesto & Vila Isabel \\
14 & Hospital Universitário Clementino Fraga & Cidade Universitária \\
15 & Hospital Municipal Miguel Couto & Gávea \\
16 & Hospital Universitário Gaffrée e Guinle & Tijuca \\
17 & Hospital da Lagoa & Lagoa \\
18 & Hospital Geral de Ipanema & Ipanema \\
19 & Hospital dos Servidores do Estado & Saúde \\
20 & Hospital Municipal Paulino Werneck & Ilha do Governador \\
21 & Hospital Municipal Souza Aguiar & Centro \\
\hline & & \\
\hline
\end{tabular}

pelos diagramas originalmente gerados (Figura 2). A partir desses valores, foram construídas as circunferências ponderadas. Quando o ponto gerador está contido na dominância estudada - ou seja, a circunferência construída contém o ponto em sua área - realiza-se a intersecção de polígonos. Em caso contrário - quando o ponto está fora da circunferência - sua dominância é considerada toda a área restante. Assim foram construídas as áreas de abrangência, obtendo-se, ao final, um mapa com os pontos dos hospitais e suas áreas respectivas.

\section{Resultados}

A seguir tem-se o mapa com as áreas de abrangência construídas a partir do Diagrama de Voronoi Clássico, apresentando, como pontos geradores, os hospitais gerais públicos (Figura 2). Seus códigos podem ser vistos na Tabela 1. A Figura 3 mostra o mapa completo com os pontos geradores e as áreas definidas pelo Diagrama de Voronoi Ponderado.

\section{Discussão e conclusão}

Na Figura 2 pode-se observar a distribuição geográfica das unidades hospitalares estudadas com maior concentração nas regiões Centro-Sul e Norte do município (lado direito do mapa), totalizando, nessa área, 13 hospitais, mais da metade das unidades. Essa concentração está relacionada ao crescimento habitacional da cidade e, também, ao processo original de construção desses hospitais (Oliveira \& Teixeira, 1986). Em decorrência dessa concentração, ao ser construído o Diagrama de Voronoi Clássico produz-se, na região central, grande número de polígonos com áreas de abrangência pequenas em comparação à região Norte e Oeste da cidade (esquerda no mapa). Esse problema é reduzido pelo uso do Diagrama de Voronoi Ponderado, pois, além da distância - empregada na construção dos polígonos na forma clássica -, outro fator é considerado: a oferta de internações dos hospitais. Efetuando-se o cotejo entre os dois mapas (Figuras 2 e 3), essas diferenças ficam bem nítidas nas Zonas Central, Norte e Sul (lado direito dos mapas), onde, estabelecendo-se a divisão por área de planejamento, são encontrados cinco setores, 
Tabela 2

Unidades, totais de internação em um ano e respectivos pesos estimados.

\begin{tabular}{lcc}
\hline Unidade & Total & $\%$ (W) \\
\hline Hospital Universitário Clementino Fraga & 26.900 & 9,1 \\
Hospital Universitário Pedro Ernesto & 25.493 & 8,6 \\
Hospital Geral de Bonsucesso & 23.410 & 7,9 \\
Hospital Municipal Souza Aguiar & 22.984 & 7,7 \\
Hospital Municipal Miguel Couto & 21.219 & 7,2 \\
Hospital Estadual Getúlio Vargas & 19.281 & 6,5 \\
Hospital Estadual Pedro Il & 19.150 & 6,5 \\
Hospital dos Servidores do Estado & 17.658 & 6,0 \\
Hospital Universitário Gaffrée e Guinle & 15.478 & 5,2 \\
Hospital Municipal Salgado Filho & 15.204 & 5,1 \\
Hospital Estadual Rocha Faria & 13.125 & 4,4 \\
Hospital Estadual Carlos Chagas & 11.175 & 3,8 \\
Hospital Geral do Andaraí & 11.035 & 3,7 \\
Hospital Estadual Albert Schweitzer & 10.604 & 3,6 \\
Hospital Geral de Jacarepaguá & 8.230 & 2,8 \\
Hospital da Lagoa & 7.250 & 2,4 \\
Hospital Raphael de Paula Souza & 7.175 & 2,4 \\
Hospital Geral da Piedade & 7.130 & 2,4 \\
Hospital Geral de Ipanema & 5.840 & 2,0 \\
Hospital Municipal Lourenço Jorge & 5.234 & 1,8 \\
Hospital Municipal Paulino Werneck & 3.029 & 1,0 \\
Total & 296.604 & 100,0 \\
\hline For Cad & &
\end{tabular}

Fonte: Cadastro de Hospitais do DATASUS.

e, com o Diagrama de Voronoi Clássico, doze setores. Tal resultado sugere que o número e o tamanho das APs utilizadas pela SMS-RJ poderiam ser redefinidos para fins de planejamento em saúde. Porém, um dos problemas que surgiram com o uso desta técnica foi a criação de áreas de mesmo tamanho para pontos com poder de atração e, portanto, com pesos diferentes. Por exemplo, o Hospital de Ipanema (18) e o Hospital Municipal Miguel Couto (15) foram definidos como possuindo áreas grandes e, aproximadamente, de mesmo tamanho, apesar de oferecerem serviços diferenciados à população, da mesma forma que número de leitos marcadamente diferentes. Outro caso é a área definida para o Hospital Municipal Paulino Werneck (20) em comparação à do Hospital Universitário Pedro Ernesto (13), ambas de tamanhos semelhantes, ainda que o hospital universitário também ofereça serviços de atendimento diferenciados e maior número de leitos.

No Diagrama de Voronoi Ponderado (Figura 3), as áreas produzidas têm igualmente tamanhos diferenciados, porém não mais definidas por retas, mas por arcos de circunferências. Como se pode observar, as discrepâncias cita- das são minimizadas. Por exemplo, os polígonos gerados pelos pontos 20 e 13, que apresentavam tamanhos semelhantes, sofrem modificações. A área definida pelo ponto 20 diminui sensivelmente, e o ponto 13 fica sem área de abrangência definida. $\mathrm{O}$ mesmo acontece com o de número 18, que tem sua área de abrangência reduzida.

Um fato que a literatura coloca como possível é verificado na produção das áreas dos pontos 5 (Hospital Estadual Carlos Chagas), 7 (Hospital Geral de Jacarepaguá) e 8 (Hospital da Piedade), onde é criada uma área não contida em nenhum dos polígonos vizinhos, ou seja, área não atendida por nenhum hospital ao seu redor, possivelmente atendida por hospital que tenha, como área de abrangência, todo o plano de estudo (Boots, 1980; Aurenhammer \& Edelsbrunner, 1984). Da mesma forma, outra pequena área, ao sul do município e próxima à costa, resultou na mesma condição - não contida por seus pontos vizinhos.

Os pontos que ficaram sem definição precisa de suas áreas de abrangência foram o 15 (Hospital Municipal Miguel Couto), o 21 (Hospital Municipal Souza Aguiar) e o 14 (Hospital 


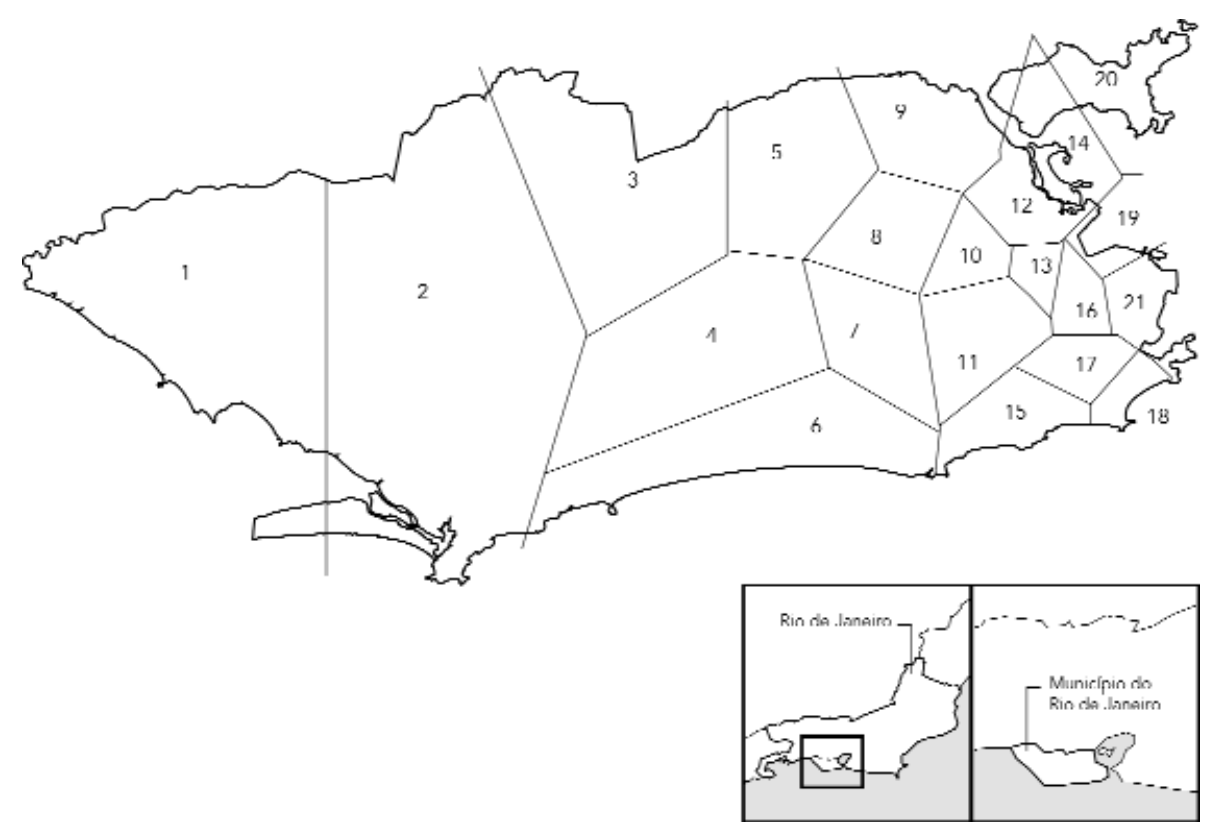

Figura 3

Áreas de Abrangência construídas pelo Diagrama de Voronoi Ponderado e Hospitais Gerais do Município do Rio de Janeiro.

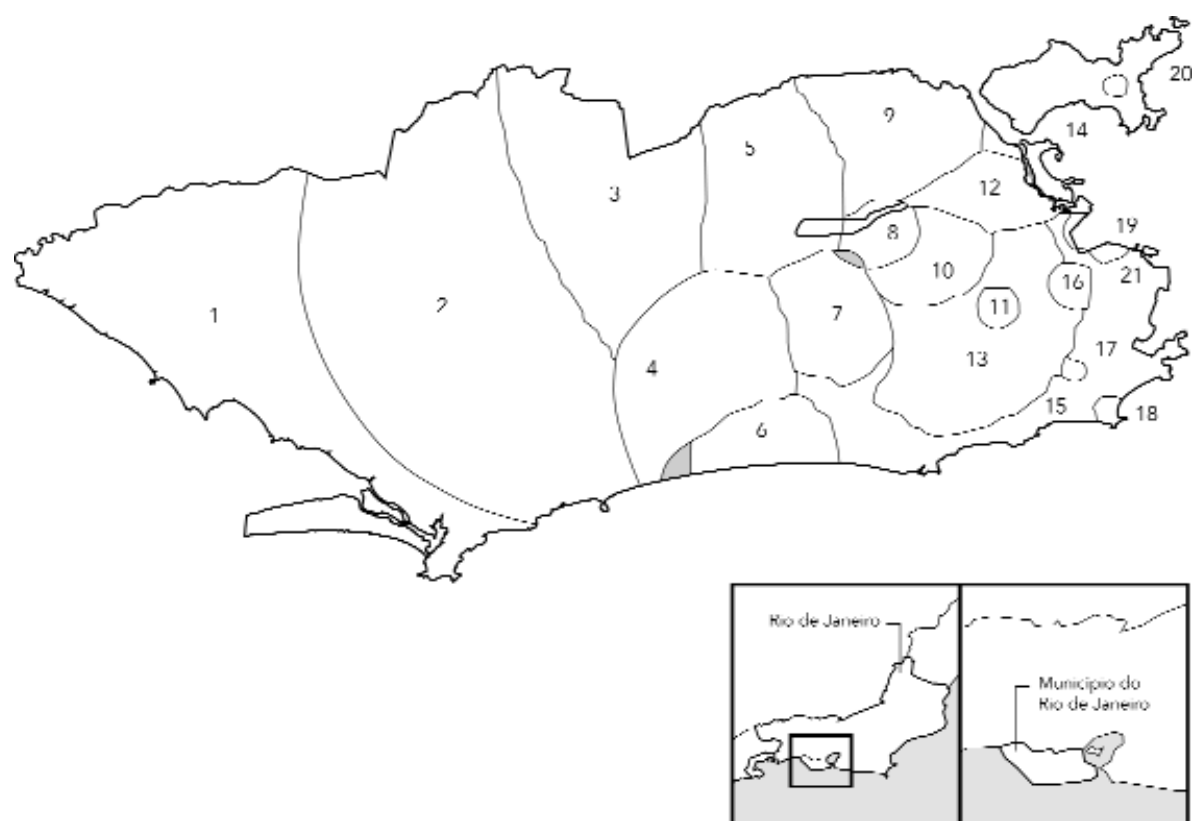


Universitário Clementino Fraga). Em relação a esse último, ao sobrepor-se o Diagrama Ponderado ao mapa do Município do Rio de Janeiro, observa-se que a sua área de abrangência corresponde principalmente à região da Ilha do Fundão, Ilha do Governador e Ramos. Os outros dois pontos possuem áreas comuns, como observado nas regiões Sul e Centro do município (à direita no mapa, Figura 3). Em termos de atendimento, é possível afirmar que esses hospitais seriam responsáveis por aquelas áreas, mencionadas acima, aparentemente sem cobertura.

Uma das vantagens da aplicação do Diagrama de Voronoi (Clássico ou Ponderado) à definição de áreas de abrangência para unidades de saúde é a acessibilidade dos dados para seu cálculo, com exceção dos mapas, que devem ser digitalizados. Já existem informações, ao nível municipal, a respeito do número de leitos, de consultórios e de profissionais de cada estabelecimento de saúde ligado ao SUS, as quais são necessárias para definir-se o poder de atração de dada unidade. O método também possui a vantagem de possibilitar uma divisão geográfica em saúde que não recorre a critérios geopolíticos ou empíricos. Por exemplo, outras metodologias, como a freqüentemente empregada definição de um raio de abrangência para uma unidade, resultam, muitas vezes, excessivamente arbitrárias, já que não há parâmetros bem definidos para o cálculo desse raio em determinada unidade de saúde.

Como desvantagem pode ser citada a dificuldade de obtenção de mapas digitalizados em municípios ou, quando estes já existem, mapas com unidades de saúde igualmente georeferenciadas. Outra desvantagem é que redes de trânsito, barreiras geográficas e urbanas nem sempre são facilmente incorporadas à construção dos polígonos, o que pode acarretar áreas de abrangência de baixa praticidade, como, por exemplo, aquelas cortadas por estrada. No presente estudo, isto pode ser ilustrado com o exemplo da Avenida Brasil, via pública que cruza o município da região Central à região Oeste, dificultando, com isso, o acesso a alguns hospitais, como o Estadual Rocha Faria (2), o Estadual Albert Schweitzer (3), o Estadual Carlos Chagas (5), o Geral de Bonsucesso (12) e o Universitário Clementino Fraga (14).

O método pode ser utilizado, além disso, de modo a respeitar os diferentes níveis de hierarquia do sistema. Por exemplo, existe a possibilidade de construir o Diagrama de Voronoi, em particular, para postos de saúde, centros de saúde ou mesmo hospitais especializados. Além disso, as áreas de abrangência têm ainda a possibilidade de serem reajustadas de acordo com outros parâmetros, tais como densidade demográfica da região ou prevalência de agravo específico.

Finalmente, após a construção das áreas de abrangência, torna-se possível o cálculo de população adscrita para cada unidade de saúde, empregando-se, para isto, os setores censitários e mapas dos Diagramas de Voronoi Ponderado. Manualmente ou mediante programas, é preciso então descobrir quais setores estão contidos em cada polígono. Com isto, podem ser feitos estudos com toda a gama de dados oriundos dos censos populacionais realizados pelo IBGE, direcionando a definição de áreas de abrangência para as unidades básicas de saúde e possibilitando estudos de cobertura e resolutividade, o que acarretará melhor alocação e utilização de recursos.

\section{Agradecimentos}

Nossos agradecimentos à FAPERJ, que proporcionou bolsa de Mestrado a um dos autores (FAVSR) e ao apoio FINEP/ CNPqMCT (Projeto PRONEX Centros de Excelência 4196093700). 


\section{Referências}

AURENHAMMER, F. \& EDELSBRUNNER, H., 1984. An optimal algorithm for constructing the weighted Voronoi diagram in the plane. Pattern Recognition, 17:251-257.

BOOTS, B. N., 1980. Weighting Thiessen Polygons. Economic Geography, 56:248-259.

BOOTS, B. N.; OKABE, A. \& KOKICHI, S., 1995. Spatial Tessellations: Concepts and Applications of Voronoi Diagrams. England: John Wiley and Sons Ltd.

BOWYER, A., 1981. Computing dirichlet tesselations. Computer Journal, 24:162-166.

BRASSEL, K. E. \& REIF, D., 1979. A procedure to generate Thiessen polygons. Geographical Analysis, 11:289-303.

BULLEN, N.; MOON, G. \& JONES, K., 1996. Defining localities for health planning: A GIS approach. Social Science and Medicine, 42: 801-816.

CLARKE, K. C.; McLAFFERTY, S. L. \& TEMPALSKI, B. J., 1996. On epidemiology and Geographic Information Systems: A review and discussion of future directions. Emerging Infectious Diseases, 2: 85-92.

GIOVANELLA, L. \& FLEURY, S., 1996. Universalidade da atenção à saúde: Acesso como categoria de análise. In: Política de Saúde: O Público e o Privado (C. Eibenschutz, ed.), pp. 177-198, Rio de Janeiro: Editora Fiocruz.

GREEN, P. J. \& SIBSON, R., 1977. Computing Dirichlet tesselations in the plane. Computer Journal, 21: 168-173.

IBGE (Fundação Instituto Brasileiro de Geografia e Estatística), 1994. Anuário Estatístico Brasileiro. Rio de Janeiro: IBGE.

KOHLI, S.; SAHLÉN, K.; SIVERTUN, Å.; LOFMAN, O.; FOLDEVI, M.; TRELL, E. \& WIGERTZ, O., 1997. Individuals living in areas with high background radon: A GIS method to identify populations at risk. Computer Methods and Programs in Biomedicine, 53:105-112.

LEE, D. T. \& DRYSDALE, R. L., 1981. Generalization of Voronoi Diagrams in the plane. SIAM Journal Computers, 10:73-87.

LOVE, D. \& LINDQUIST, P., 1995. The geographical accessibility of hospitals to the aged: A Geographic Information Systems Analysis within Illinois. Health Services Research, 29:629-651.

MACEDONIO, G. \& PARESCHI, M. T., 1991. An algorithm for the triangulation of arbitrarily distributed points: Applications to volume estimate and terrain fitting. Computers and Geosciences, 17: 859-874
MARTIN, D. \& WILLIAMS, H. C. W. L., 1992. Marketarea analysis and accessibility to primary healthcare centers. Environment and Planning A, 24: 1009-1019.

MS (Ministério da Saúde), 1982. Anexo à Portaria no 3046/82: Parâmetros para Planejamento Assistencial a serem Utilizados pelo INAMPS. Brasília: MS.

MS (Ministério da Saúde), 1997. Movimento de Autorização de Internação Hospitalar. CD-ROM. Brasília: Departamento de Informática do SUS, Fundação Nacional de Saúde, MS.

NIJKAMP, P. \& SCHOLTEN, H. J., 1993. Spatial Information Systems: Design, modeling and use in planning. International Journal of Geographical Information Systems, 7:85-96.

OLIVEIRA, J. A. A. \& TEIXEIRA, S. M. F., 1986. (Im)Previdência Social - 60 Anos de História da Previdência. Petrópolis: Editora Vozes.

RHYNSBURGER, D., 1973. Analytic delineation of Thiessen Polygons. Geographical Analysis, 5:133144.

SALMELA, R., 1993. Regional inequalities in health and health care in Finland and Norway. Health Policy, 24:83-94.

SIBSON, R., 1980. The Dirichlet Tesselations as an aid in data analysis. Scandinavian Journal of Statistics, 7:14-20.

TSAI, V. J. D., 1993. Fast topological construction of Delaunay Triangulations and Voronoi Diagrams. Computers and Geosciences, 19:1463-1474.

UNGLERT, C. V. S., 1990. O enfoque da acessibilidade no planejamento da localização e dimensão de serviços de saúde. Revista de Saúde Pública, 24: 445-452.

UNGLERT, C. V. S.; ROSENBURG, C. P. \& JUNQUEIRA, C. B., 1987. Acesso aos serviços de saúde: Uma abordagem de geografia em Saúde Pública. Revista de Saúde Pública, 21:439-446.

WATSON, D. F., 1981, Computing the n-dimensional Delaunay Tessellation with application to Voronoi Polytopes. Computer Journal, 24:167-172.

ZAIDI, S. A., 1994. Planning in the health sector: From whom by whom? Social Science and Medicine, 39: 1385-1393.

ZWARENSTEIN, M.; KRIGE, D. \&WOLFF, B., 1991. The use of a geographical information system for hospital catchment area research in Natal/KwaZulu. South Africa Medicine Journal, 80:497-500. 